\title{
The TEAM Project - An Update
}

\author{
U. Dahmen
}

National Center for Electron Microscopy, LBNL, University of California, Berkeley, CA 94720

Due to its unique ability to characterize nanoscale structures, electron microscopy is a key tool for nanoscience. The best instruments today provide local information with a resolution of $1 \AA$ or better from individual nanostructures. Recent advances in electron optics promise a considerable improvement in the capabilities of electron microscopes, toward a new generation of electron optical instruments, which are likely to have an important impact on our understanding of the structure of materials at the atomic level.

The TEAM project (Transmission Electron Aberration-corrected Microscope) is a collaborative effort [1] funded by the Department of Energy to construct a next-generation electron microscope based on current progress in electron optics, stages and detectors, and optimized for corrected optics and increased stability. The five partner labs collaborating in this project are shown in Figure 1. The first TEAM instrument will be installed at the National Center for Electron Microscopy in 2009 and will be operated as a user facility to support the growing need for atomic-scale characterization in the nanoscience community.

The TEAM proposal grew out of a major review of the four user facilities for electron-beam microcharacterization supported by DOE, where a vision for a collaborative project was first presented. Subsequently, the prospects for aberration correction, the need for advanced electron scattering and the opportunities for new science enabled by aberration correction were discussed in a series of workshops at ANL (2000), LBNL (2002), M\&M San Antonio (2003) and M\&M Savannah (2004) [2]. The input from the scientific community has helped shape the direction of the project, whose primary driving force has always been the scientific goal to better characterize materials rather than the development of instrumentation.

Its origin in the needs of nanoscience has imposed a set of important constraints on the project in terms of timing, goals and approach. To maximize the impact on the DOE nanoscience centers at LBNL, ANL, ORNL and BNL, an initial instrument is scheduled to be installed in 2008. Although the first TEAM instrument will be physically located at NCEM in Berkeley, it will be accessible to users by remote operation from any of the five partner labs. To be able to address materials issues across disciplines, the machine will be designed to operate between 80 and $300 \mathrm{kV}$. Full aberration correction for the probe and the image will allow $0.5 \AA$ resolution in both, the STEM and TEM modes. The post-specimen corrector is being designed to correct for spherical as well as chromatic aberration. A novel 5-axis stage driven entirely by piezoceramic elements will provide unprecedented control over sample position and tilt. This stage will facilitate data collection for high-resolution tomography. The stage will also include standardized experimental modules to allow users to design MEMS-controlled in-situ experiments for dynamic observation of materials. A 5-mm pole-piece gap will provide sufficient space for in-situ experiments and high tilt angles for tomography. The instrument will include a monochromator for $0.1 \mathrm{eV}$ energy resolution as well as an imaging energy filter. In addition, new designs for electron detectors are being considered that would allow much faster and more efficient data acquisition.

As a DOE project, TEAM is subject to a very stringent set of rules that were designed to govern major construction projects such as buildings, synchrotron or neutron scattering facilities. These rules impose strict reporting requirements with great emphasis on adherence to timelines and budgets. The five partner labs are working together in a collaborative mode that is unusual for the microscopy community but that reflects a new unity based on the overarching effect of aberration correction for all segments of the microscopy community - STEM and TEM, materials and biology, researchers in universities, industry and national labs, manufacturers and developers. Each of the five 
partner labs is responsible for delivery of a particular component, technique or expertise needed for the TEAM instrument. Thus, ANL has responsibility for the development of the post-specimen corrector on the TEM side of the instrument, in close collaboration with electron optics manufacturer CEOS in Heidelberg. ORNL is in charge of development and optimization of the probe corrector on the STEM side of the machine. FS-MRL and NCEM jointly are developing the new piezoceramic stage, and BNL together with LBNL are accountable for the development and implementation of novel electron detectors. The task of column integration is under the responsibility of LBNL, collaborating closely with the column manufacturer. Several of the sub-tasks involve operation of a test column for a period of time to shake down, align, optimize and integrate different components.

This new mode of collaboration has required an extensive series of discussion meetings over the last year to make decision vital for the project. One of the most important of these was the extent and mode of collaboration with manufacturers. Based on detailed analysis and discussion, the collaboration has selected FEI as the major partner for column integration will work with CEOS on development and implementation of aberration correctors.

This talk will give a brief overview of the TEAM collaboration with its scientific and technical goals, and present an update on recent technical developments such as stage design and requirements for tomography [3].
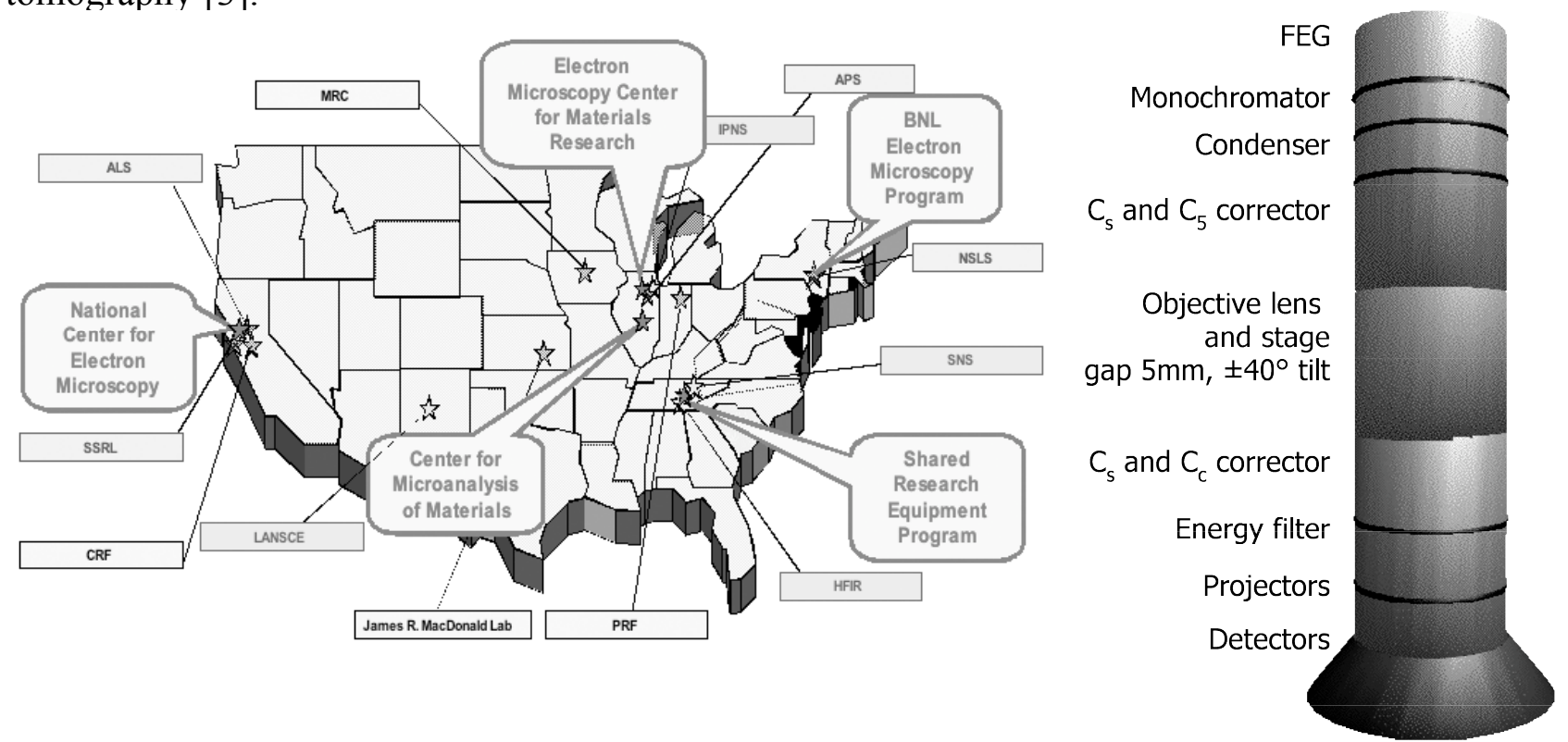

FIG.1 - Geographic distribution of the five TEAM partner labs (callouts) along with other major DOE user facilities supporting scientific research in the US.

FIG.2 - Schematic of the TEAM column illustrating major components. Each partner lab is responsible for a component, technique or expertise.

\section{References}

[1] Full list of TEAM investigators: U. Dahmen, N. Browning, C. Kisielowski, E, Stach, A. Schmid - (LBNL), D. Miller, B. Kabius, N. Zaluzec - (ANL), Y. Zhu, J. Wall - (BNL), I. Petrov, I. Robertson, R. Twesten, J.M. Zuo - (FS-MRL), I. Anderson, J. Bentley, S. Pennycook - (ORNL) [2] Reports from these workshops and more information on the TEAM project can be found at http://ncem.lbl.gov/team3.htm

[3] The TEAM project is supported by the Director, Office of Science, Office of Basic Energy Sciences, Division of Materials Sciences and Engineering, of the U.S. Department of Energy under Contract No. DE-AC03-76SF00098. 